\title{
Orbital Fractures - An Overview
}

\author{
Pavan Kumar B ${ }^{1}$, YashwanthYadav², Brahmaji Rao ${ }^{3}$, Haripriya Chari ${ }^{4}$
}

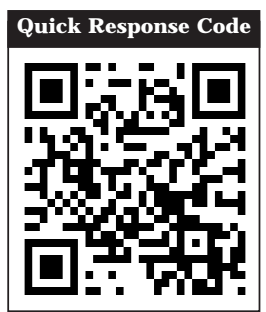

doi: $10.5866 / 2017.9 .10040$

${ }^{1}$ Professor \& HOD

2,4Senior Lecturer

${ }^{3}$ Professor

Department of Oral \& Maxillofacial Surgery,

Kamineni Institute of Dental Sciences,

Narketpally,Nalgonda, Telangana State.

\section{Article Info:}

Received: J anuary 12, 2017

Review Completed: February 13, 2017

Accepted: March 10, 2017

Available Online: March, 2017 (www.nacd.in)

(c) NAD, 2017 - All rights reserved

Email for correspondence:

pavankumarbatchu40@gmail.com

\begin{abstract}
:
Abstract: Of thevarious maxill ofacial fractures, orbital fractures involve one of the most complex anatomy resulting in a variety of both esthetic and functional disturbances, often associated with other complex fractures. The opinion on the management of such fractures, especially when involving soft tissue, is in itself varied and controversial. We aim to provide an overview of the various types of orbital fractures, their diagnosis, the anatomical and surgical factors involved, and the reconstructive options available for the management of orbital trauma.
\end{abstract}

Key words: Orbit, blow out, trap door, optic nerve

\section{Introduction:}

The orbital skeleton represents an important anatomic crossroad because of its intimate relationship to the central nervous system, the nose, the para nasal sinuses, the face and the structures related to the support and function of the eye. As such it is of clinical and surgical significanceto many disciplines. The nature of orbital injuries has evolved from the early centuries, when they were often associated with war and assault with a high incidence of mortality and morbidity, to the later part of $20^{\text {th }}$ century, when far more complicated injuries were seen, especially with the escalation in the number of automobile accidents and automotive trends towards faster vehicles and violent crimes. ${ }^{1}$ There has been much discussion and controversy over the indications for and timing of orbital fracture repair specially when associated with diplopia and enopthalmos. The axiom of orbital surgery is to restore the orbit to the premorbid and esthetic form with preservation of function. Failure to recognize the complexity of bone injury and secondary soft tissue sequelae has resulted in suboptimal results in atleast $1 / 3^{\text {rd }}$ of patients. ${ }^{2}$ Currently a greater understanding of the complexity of the orbital anatomy, patterns of orbital trauma and evolution of diagnostic and surgical technique have revolutionized the management of orbital trauma, making excellent results a rule rather than an exception.

\section{Anatomical Considerations:}

The orbital region can be divided into 4 basic components - the bony orbit, the periocular soft 
tissues, the globe and the protective soft tissue apparatus consisting of the lids and lacrimal apparatus.

The Bony Orbit: The osteology of the orbit consists of a union of following bones: zygoma, maxilla, lacrimal, ethmoid, frontal, palatine and sphenoid. By the age of five, orbital growth is $85 \%$ complete and it stops between seven years of age and puberty. ${ }^{3}$ The average adult or bit has a volume of $30 \mathrm{cc}$ and the globe average is 7cc. The surgeon must be aware of the limits of safe sub periosteal dissection. A subperiosteal dissection can be safely extended $25 \mathrm{~mm}$ posteriorly from the inferior and the lateral rims. An exploration distance of $30 \mathrm{~mm}$ from the superior orbital border or anterior lacrimal crest can be performed. The surgeon must bear in mind that these "safe" distance are referenced from intact adult orbital rims. When a traumatic force has displaced a portion of the rim, it is generally in a direction (i.e., posterior, medial) that effectively reduces these distances. At all times, care must be taken to avoid disruption of medial canthal tendon, lacrimal apparatus, pulley of the superior oblique muscle, supraorbital nerves and vessels, structures attach to Whitnall's tubercle, and origin of inferior oblique muscle.Rontal et al (1979) arrived at mean distance for locating vital structures in relationship to identifiable fine bony landmarks. ${ }^{3}$ (Table-1)

\section{Classification of Orbital Fratures:}

Orbital fractures occur in numerous patterns typically described by their anatomic location. While these classifications of orbital fractures are useful in communication, the assessment and treatment of each patient must be individualized.

1) CRAMER AND TOOZE (1965) classified the orbital floor injuries based on the surgical anatomy, emphasizing that multiple facial injuries can accompany the blow out fractures. In an effort to clarify the situation further they refer to them as pure or impure blow out fractures. ${ }^{4}$

1. Pure blow-out fractures

Punched out piece of bone

Hinged bony fragment

Linear fracture incarcerating muscle

Fragment penetrating inferior rectus

2. Impure blow out fracture

Comminuted orbital rim
Lefort II

Incontinuity fracture- Nasal, Ethmoid, Zygoma Discontinuity fracture - Nasal, Zygoma

3. Blow out apparent only after open reduction of fracture dislocation zygoma

4. Comminuted floor, no incarcination - Floor present but sagging into antrum, Floor nearly completely absent

2. CONVERSE J M, SMITH B, OBEAR MF, WOOD SMITH D (1967) classified the orbital fractures to clarify that not every fracture of the floor of the orbit is a blowout fracture and the term blow out fracture defines a mechanism of fracture and does not apply to all orbital fractures. ${ }^{5}$

\section{Orbital Blow-out Fractures}

A. Pure blow-out fractures - Fractures through the thin areas of the orbital floor, medial and lateral wall. The orbital rim is intact.

B. I mpure blow-out fractures- Fractures associated with fracture of adjacent facial bones. The thick orbital rim is fractured and its backward displacement causes a comminution of the orbital floor, the posterior displacement of the orbital rim permits traumatizing force to be applied against the orbital contents, which produces a superimposed blow-out fractures.

\section{Orbital fractures blow-out fracture}

A. Linear fractures, in upper maxillary and zygomatic fractures. These fractures are often uncomplicated from the stand point of the orbit.

B. Comminuted fracture of the orbital floor with prolapse of the orbital contents into the maxillary sinus is often associated with fracture of midfacial bones.

C. Fracture of thezygoma with fronto-zygomatic separation and downward displacement of zygomatic portion of the orbital floor and of the lateral attachment of the suspensory ligament of lock wood.

3. According to Rowe and Williams (1994) orbital fractures are classified as:

I. Zygomatic complex fractures

A. Fractures stable after el evation

a) Arch only (medially displaced)

b) Rotation around the vertical axis Medially, Laterally 
B. Fractures unstable after elevation
a) Arch only (inferiorly displaced)
b) Rotation around the horizontal axis
- Medially
- Laterally
c) Dislocation en bloc
-I nferiorly
-Medially
-Postero laterally
d) Comminuted fractures

II. I solated fractures of the orbital rim
A. Superior rim
-Lateral third (Lacrimal recess)
-Central third (Supra orbital nerve)
-Medial third (Frontal sinus)
B. Inferior rim
-Central third (I nfra orbital nerve)
- Medial third (Inferior oblique origin)
C. Medial rim
-Medial canthal ligament
-Lacrimal passages
D. Lateral rim
- Lateral canthal ligament
- Suspensory ligament

III. I solated fractures of the orbital walls
A. Roof
-Anterior fossa
-Levatorpal pebraesuperioris / superior rectus
-Frontal sinus
B. Floor
-Antrum
-Infra-orbital nerve and vessels
-I nferior rectus/ inferior oblique
C. Medial wall
- Lacrimal sac and nasolacrimal canal

-Ethmoidal sinus

- Medial rectus

- Suspensory ligament

D. Lateral wall

- Superior orbital fissure and associated structures

IV. Complex comminuted fractures

\section{According to Fonseca RJ (2000)7 fractures involving orbit are classified as}

A. Zygomatic complex fracture

According to J ackson (1989) zygomatic complex fractures are classified as:

Type I - Non displaced

Type II - Segmental fracture of orbital rim

Type III - Tripod fracture

Type IV - Fragmented

B. Naso orbital ethmoid fractures

C. Internal orbital fractures

-Linear fracture (maintain periosteal attachment)

-Blow-out fracture (limited to one wall with a defect of less than $2 \mathrm{~cm}$ in diameter)

-Complex (affect two or more orbital walls, often associated with fractures of the facial skeleton outside the orbital frame such as Lefort II, III fracture or frontal sinus fracture, classified as combined fractures.)

\section{Clinical Features:}

The clinical manifestation of an orbital fracture will vary according to the interval which has elapsed since the injury, its severity and the extent to which the respective rims and walls are involved. ${ }^{8}$ Attention must be directed towards the following signs and symptoms:-

1. The peri-orbital tissues- Oedema, Circumorbital ecchymosis, Subconjuctival haemorrhage, Surgical emphysema

2. The eyelids - Abnormality of the palpebral fissure, Height, Width, Inclination, Mobility (ptosis, pseudoptosis), Integrity of margins and tarsal plates. 
3. The ligaments - Alteration in the canthal level, Alteration in the ocular level, Increased intercanthal distance (telecanthus)

4. The eye - Preservation of vision, Limitation of ocular movements, Presence of diplopia, Exophthalmos or enopthalmos, Pupillary reflexes, Ophthalmic injuries, Increased interpupillary distance, Deepening of supracanthal fold.

5. The lacrimal apparatus - Wounds involving the puncta, Injuries which might involve the passages, Epiphora

6. Neurological deficits - Paresthesia of infraorbital , supra-orbital or supra-trochlear nerves, Paresis of the extra-ocular muscles, Paresis of the facial nerve

7. The orbit - Pain on palpation and /or deformity at the superior margin, the fronto-zygomatic suture, inferior margin, the naso-frontal and naso-maxillary sutures, thezygomatic boneand/ or arch and, intra-orally, thezygomatic buttress, the lateral antral wall.

\section{Orbital Blow-Out Fractures:}

The term blow out fracture was proposed by Smith and Regan (1957). ${ }^{9}$ Willam Lang (1889) first described a depressed fracture of the orbital floor and associated condition of post traumatic enophthalmos. ${ }^{10} \mathrm{~A}$ blow out fracture is caused by the application of the traumatic force to rim of the soft tissues of the orbit generally assumed to be accompanied by a sudden increase in intra-orbital pressure. Blow out fractures result a when a blunt object larger in diameter than the orbital rim strikes the rim and orbital content simultaneously. Object smaller than orbital aperture are much more likely to penetrate or rupture the globe and orbital contents.

\section{Mechanism of orbital blow-out fracture:}

Langrange and Leforte had thought that orbital fractures were produced by force transmitted from the orbital rim to orbital floor. ${ }^{11}$ Raymold Pfeiffer studying 140 orbital fractures, said, 'it is evident that force of the blow received by the eye ball was transmitted by it to the walls of the orbit with fractures of more delicate portion. ${ }^{12}$ Smith and Regan (1957) defined the term 'blow-out', fracture of the orbital floor caused by sudden increase in intra-orbital or hydraulic pressure. ${ }^{9}$ They demonstrated the mechanism experimentally in which a blow to the eyeball caused fracture of the orbital floor, where as blows to the rim on the contralateral side caused no fracture.

J ones and Evans (1967), noted that the globe was closer to the medial wall and floor than it was to the other walls and proposed that the globe established the fracture by being driven back along the access of the orbit, striking the orbital walls when the size of the globe prevented further posterior displacement. The most common site of blow out fracture was in thefloor medial to theinfraorbital nerve in the posterior portion of the orbit. ${ }^{13}$

Reny and Stricker (1969) suggested the following hypothesis. A traumatic force striking the inferior orbital rim, which is sufficiently resilient to transmit the force to the orbital floor, fractures the latter while the rim rebounds without fracturing. ${ }^{14}$

\section{Orbital Blow-In Fractures:}

The volume occupied by the soft tissue contents (the eye and adnexa) may expand or contract secondary to the direction of the orbital fracture displacement. Orbital blow-in fracture has been used to describe the orbital volume concentration that occur secondary to some types of bone displacement.Orbital blow-in fractures comprise a distinct clinical entity characterized by displacement of fracture fragments into the orbital cavity with a consequent diminution in the intra-orbital volume. ${ }^{15}$ Soft tissue contents are displaced forward and out of the orbital cavity, resulting in ocular proptosis. Dingman and Natwig (1964) first described the condition in a patient with a superiorly displaced fracture of the orbital floor.$^{16}$ Since then isolated cases of blow-in fractures have been documented involving the floor, lateral rim, zygoma and roof of the orbit. ${ }^{17-20} \mathrm{~A}$ typical fracture causing orbital contraction is an orbital roof fracture in which the displacement of the roof downwards and backwards results in downward and forward displacement of the globe.

\section{Mechanism}

Orbital bow-in fractures generally results from high velocity trauma. Lighterman and Reckson (1979) speculated that a growing hematoma and increased air pressure trapped in a maxillary sinus by a ball valve mechanism maintained an elevated floor fragment within the orbital cavity. ${ }^{17}$ According 
to Rowe and William (1994), it is unlikely that compression of the air within the antrum can be responsible for blowing fractures since there is means of escape through the ostium. ${ }^{6}$ They found that the bone is fractured by linear shock wave and pulled up by negative pressure induced when the globe and periorbital fat rebound after impact.

\section{Imaging:}

Plane radiographs for the diagnosis of orbital injuries include water's, Caldwell, Towne's, bilateral lateral oblique and lateral head view.

Ultrasound: UItrasound represents a safe, inexpensive, non-invasive, portable and readily available diagnostic imaging modality. It may be indicated in the diagnosis of isolated orbital trauma or in the polytraumatized patients in whom positioning difficulties exists. It is contraindicated in suspected penetrating injuries to the globe to prevent application of pressure to the traumatized globe in order to minimize the possibility of causing expulsion of the intraocular contents from an occult ruptured globe and is not always feasible because of soft tissue swelling, laceration of the eyelids or pain. Medial floor blow out fractures are often missed on ultrasound. The use of curved array scanner showed satisfying results in the investigation of orbital floor, infraorbital rim and lateral orbital walls. ${ }^{21}$

Computerized Tomography: For evaluating injury in the complex anatomical regions such as an orbits, nasofrontal ducts, and nasal cavity, $2 \mathrm{~mm}$ thick slices are required for detailed examination. CT scan is also valuable in evaluating the globes and foramen. Optic nerve compression secondary to bony fragment displacement is usually visualized only on CT scanning.

Magnetic Resonanace I maging: MR imaging is not applicable for the initial assessment of orbital injuries in the setting of trauma. MR imaging is the radiologic examination of choice for detection of wood foreign bodies. MR imaging better differentiates hematomas from edema, and is hel pful in assessing vascular injuries such as carotid cavernous sinus fistulas or post traumatic pseudo aneurysms.

\section{Surgical Management of Orbital Fractures:}

This involves an exploration of the intact bone and defining the location by surgical dissection of the intact bone around the orbital defect. Identification of intact bone landmarks permits a plan for reconstruction of the bony wall of the orbit in its anatomic position. Other goals for the treatment of orbital blow-out fractures include:

a. Disengage entrapped midface ligamental structures and restore ocular rotatory function.

b. Replace orbital contents into the usual confines of the normal bony orbital cavity, including restoration of orbital volume and shape.

c. Restore orbital cavity walls, which in effect places the tissues into their proper position and dictates the shape into which the soft tissue can scar.

Timing: In isolated blow out fractures, it is not necessary to operate immediately unless muscle laceration or severe restriction of vision is present. In children, delay of operation is not desirable because bony regeneration is rapid and osteotomy will be required, and freeing of incarcerated orbital soft tissue contents then become less effective. Late motility problems from significant incarceration persists if treatment is postponed for 2 to 3 weeks despite the late release of orbital contents. I mmediate intervention in treatment of orbital floor fracture is also indicted when orbital soft tissue entrapment generates the oculo-cardiac reflex. ${ }^{19}$

Surgical Techniques: A number of methods have been advocated for treatment of blow-out fractures. Thesurgical approach has involved either the eyelid or the canine fossa through the maxillary sinus and recently, endoscope procedures. The eyelid or conjuctival approach to the orbital floor is preferred because it facilitates the disengagement of any entrapped or prolapsed orbital tissues under direct vision.

a) Support of orbital floor from maxillary antrum: For antral support the defect should not exceed $5 \mathrm{~mm}$ in diameter, any prolapsed tissue should be replaceable, they should be no adhesions restricting ocular mobility, theantral floor should be stable and the orbital margins should be intact or reconstituted. Antralballoons: $f$ a special antral balloon is not available, it is possible to use a $30 \mathrm{ml}$ Foley's catheter as originally suggested by J ackson et al (1956). Fluid under pressure exerts an even force the whole surface of the balloon so that the selective support of an area is not achieved with the degree of control obtained by an antral pack. 
b) Endoscopic approaches: Endoscopic approaches through the maxillary sinus have permitted direct visualization of the orbital floor, manipulation of soft tissueand floor repair by avoiding and eyelid incision.

c) Exposure of orbital floor: Access to the inferior orbital margin and the orbital floor may be gained by the transcutaneous and transconjunctival approaches. The major difference between cutaneous incision is the level at which the incision is placed in the skin of the eyelid and the level at which the muscle is transected to expose the orbital septum/ periosteum. The orbital rim incision is indicated in the presence of marked periorbital oedema that precludes canthotomy and impedes identification or placement of higher lid incisions.

II. Restoration of Continuity of Orbital Floor: Restoration of continuity of orbital floor is required in all orbital floor fractures, except in small fractures in which the entrapped structures can be freed readily and the forced duction test shows that the free rotation of eyeball has been re-established. Therefore, reconstruction of the orbital floor fracture is required when it demonstrates a bone defect or a malpositioned, comminuted, or weekend orbital floor.The functions of the orbital implant are to seal off the antral cavity from the orbit, to provide a physiologically acceptable and physically inert smooth surface which will not form adhesions, to restore the contour and dimensions of the orbit, to provide some indirect support for the globe.

Autogenous materials: Autogenous tissues were the first material used to reconstruct the internal orbit and remain a frequently used material today.

Autogenous bone: Endochondral and membranous bone surface are used in the orbital reconstruction with the major donor sites for each being ileac crest and calvarium.

Autogenous cartilage: Proponents of the autogenous cartilage tout its ease of harvest, flexibility and limited donor site morbidity as its main advantages.

Allogenic materials: Allogenic materials (allografts, homografts) and xenografts contains no living cells but, depending on the material, may causes osteoinductive and/or osteoconductive properties. These materials become incorporated into post tissues by providing a structural framework for ingrowth of the host tissues. They do not require a second operative site; therefore they require less operative time and are generally abundant in supply.

Lyophilized fascia: Lyophilized dura and lyophilized tensor fascia lata are the two major sources of lyophilized fascia. It is readily available and easy to handle for the placement in the orbit.

Xenografts: They are the bone grafts taken from the other species. Xenografts are rarely used for the repair of internal orbital fractures. Only a few case reports are available in literature regarding their application within the orbit. Lyophilized procine dermis has been used for the repair of small defects.

\section{Non Resorbable Alloplasts:}

Metallic mesh: Disadvantages of metal alloys include the risk of extrusion and infection, and the theor etical risk to the tissues of the orbital apex from another blow to the orbit. Removal of these materials when indicated may be extremely difficult because of fibrous ingrowth through holes machined into them and also the possibility of osseous overgrowth or osseo integration of the material. Presence of metal plates may lead to inflammation and adhesions that would contribute to ocular muscle restrictions.

High Density Porous Polyethylene: High density porous polyethylene (HDPE) is commercially available as Medpor. It is highly biocompatible and processed specifically to include and control poresize (100-200 um). ${ }^{23}$ It is insoluble in tissue fluids, does not resorb or degenerate, incites minimal surrounding soft tissue reaction and possesses high tensile strength. Tissue ingrowth and formation of a mucosal lining occur even when the implant is placed over an open maxillary sinus. ${ }^{24}$

Hydroxyapatite: HA is highly biocompatible and cause minimal inflammatory reaction in the surrounding tissues. HA produces a strong mechanical bond with host bone and allows ingrowth of host tissue, providing a scaffold for bone repair. HA shows limited resorption and obviates the need for a second surgical site. HA has a minimal infection rate of $2.7 \%$ for craniofacial reconstruction. ${ }^{25-26}$.

Silicones and Polytetrafluoroethylene (Teflon): The current use of these materials is 
limited because of numerous reports of late complications arising as many as 20 years postoperatively. ${ }^{27}$ It is chemically inert with no known solvent, noncarcinogenic and able to be sterilized. It is available in felt like sheet that is easily cut to size. The majority of complications associated with silicone implants can probably be attributed to the lack of stabilization of the implants that were characteristic of early techniques.

Resorbable Alloplastics are Polylactide, Lactosorb, Polyglactin 910, Polydioxanone (Pds)

Gelatin Film: The purpose of orbital floor replacement, whether a bone graft or an inorganic implant, is to re-establish the size and shape of the orbital cavity. The orbital implant must conform to the proper contour of the floor and not provide a place where the dead place causes fluid accumulation. The failure to reconstruct the proper size and shape of the bony orbital cavity is the most frequent cause of enopthalmos. Orbital reconstruction for two or more adjacent missing walls is most easily accomplished with material that can reconstruct, in a stable fashion, the buttress supports the orbit, metal meshes, bone graft stabilized by plates, or specialized plates designed for the orbit make this difficult task straight forward. Before the closure of the wound, a forcedduction test should be performed to identify possible impingement of the reconstructive material on extra ocular muscles or their surrounding attachments.The treatment of the orbital fracture must be performed in conjunction with the reduction and fixation of fracture of the orbital rim and other bones of the midfacial area. The only technique that can adequately restore the continuity of displaced or fragmented bones is based on the full exploration of usual sites of fracture, including the medial, inferior and lateral orbit. This is best done by direct exposure of the fractured area and bone graft reconstruction of the missing portions of the orbit in conjunction with reduction of the rim fractures.

\section{COMPLICATIONS:}

Various complications following orbital trauma are encountered. It is ther efore of utmost importance to establish a clearly documented presurgical baseline as to the trauma victim's vision, ocular mobility, degree of enophthalmos, diplopia, pupillary reflex, and the appearance of the retina. In the treatment of periorbital fractures, preservation of vision takes precedence over restoration of the bony anatomy, gaze or enophthalmos. Certain complications can lead to vision loss and must be recognizedearly. These include:

1. Optic nerve compression

2. Central retinal artery occlusion

3. Retrobulbar hemorrhage

4. Superior orbital fissure syndrome

5. Cavernous sinus syndrome

6. Orbital apex syndrome

7. Intraocular injuries

\section{LATE COMPLICATIONS OF ORBIATL FRACTURE :}

1. Ectropion

2. Entropion

3. Infraorbital nerve paresthesia

4. Cerebrospinal fluid rhinorrhea

\section{CONCLUSION:}

Orbital fractures may occur as isolated fractures of the internal orbit or may involve both the internal orbit and the orbital rim. Diagnosis of a blow-out fracture is made on the basis of the characteristic clinical findings of, diplopia due to limitation of

Table 1:

\begin{tabular}{llc} 
Reference landmark & Structure & Mean distance(mm) \\
Infraorbital foramen & Midpoint of inferior orbital fissure & 24 \\
\hline Anterior lacrimal crest & Anterior ethmoidal foramen & 24 \\
\hline Anterior lacrimal crest & Optic canal(medial aspect) & 42 \\
\hline Fronto-zygomatic suture & Superior orbital fissure & 35 \\
\hline Supra-orbital notch & Superior orbital fissure & 40 \\
\hline Supra-orbital notch & Optic canal(superior aspect) & 45 \\
\hline
\end{tabular}


ocular movements, enophthalmus, orbital ecchymosis, sub-conjuctival hemorrhage, increased depth of supra tarsal fold and in some cases decreased sensation in the distribution of infra orbital nerve. The most important component of orbital reconstruction is the restoration of the pre trauma volume of the internal orbital. Materials used to reconstruct the internal orbit are both autogenous and alloplastic. Complications may result from initial trauma or from the surgical repair. Most complications arise as the result of injury such as globe and optic nerve injury, orbital hemorrhage, CSF leak, injury to nasolacrimal system, superior orbital fissure syndromeand injury to infraorbital nerve. Complications should be avoi ded by performing the surgery meticul ously and keeping a careful watch on the patient during post operative period. In conclusion, car eful preoperative interpretation of both the fracture site and the accompanying ocular signs and motility defect will enhance the chance of successful management and outcomes of orbital fracture.

\section{REFERENCES:}

1. Stephen A, Schendel, Orbital trauma: Oral and Maxillofacial Surgery Clin of North Am. W.B. Saunders.1993; 5(3).

2. Converse J M, Smith B, Wood-Smith D. Orbital and nasoorbital fractures. In converse J M (ed): Reconstructive plastic surgery, ed 2: Philadelphia, WB Saunders, 1977.

3. Rontal E, Rantal M, Guilford FT. Surgical anatomy of the Orbit. Ann Otol Rhinol Laryngol 1979; 88:382.

4. Cramer LM, Tooze FM and Lerman S. Blow out fractures of the orbit. Brit J Plastic Surgery: 171-9.

5. Converse J M, Smith B, Orbear MF, Wood-Smith D. Orbital blowout fractures: ten-year survey. Plast Reconstr Surg 1967; 39:20.

6. Rowe NL. The diagnosis periorbital injuries. Frotschritte der kiefer und Gesichtschirurgie, 1977; 22:3.

7. Ochs MW, J ohns FR. Fonscca RJ . Oral and Maxillofacial Surgery, vol 3, W.B. Saunders 2000:205.

8. Rowe NL. Fractures of the zygomatic complex and the orbit. In: Rowe NL, Williams J LI. Maxillofacial injuries. Vol 1. Edinburgh: Churchill Livingstone, 1994.

9. Smith B, Regan WF J r. Blowout fracture of orbit: mechanism and correction of internal orbital fracture. AmJ Ophthalmol 1957; 44:733.

10. Lang W. Traumatic enophthalmos with retention of perfect acuity of vision. Trans Ophthalmol Soc Engl 1889; 9:44.
11. Lagrange F. Les fractures del orbite per les proietiles deguerre,. Paris, Masson and Cie, 1917.

12. Pfeiffer RL. Traumatic enophthalmos. Arch Ophthalmol 1943; 30:718.

13. J ones E, Evans J N: 'Blowout' fractures of the orbit. An investigation into their anatomical basis. J Laryngol Otol 1967; 81:1109.

14. Reny A; Stricket M. Fracture de L'Orbite. Indications ophthalmologiques dans les Techniques operatories. Paris, Masson et cie 1969.

15. Antonyshym O, Gruss J S, Galbraith DJ et al. Complex orbital fractures: A critical analysis of immediate bone graft reconstruction. Ann Plast Surg 1989; 22:220.

16. Dingman, R.O, Natvig P. Surgery of facial fractures, Philadel phia: Saunders 1964:234.

17. .Lighterman I, Reckson C. 'Blow-in' fracture of the orbit. Ann plast. Surgery 1979; 3:572.

18. Margarone J E, Laterla orbital rim fracture. An unusual case. J Am Dent. Assoc 1982; 105 :657.

19. Goldoy J, Mathog RH. Malar fractures associated with exophthalmos. Arch Otolaryngol 1985; 111:74.

20. Flangagan J C, MCLachlan DL, Shannon GM. Orbital roof fractures: Neurological and neurosurgical considerations. Ophthalmology 1980; 87:325.

21. J ank S, Emshoff R, Etzelsdorfer M. Ultrasound versus computed tomography in the imaging of orbital floor fractures. J Oral Maxillofacial Surgery 2004; 62:150.

22. Sires BS, Stanlye RB J r., Levine LM. Oculocardiac reflex caused by orbital floor trapdoor fracture: an indication for urgent repair [letter]. Arch Ophthalmol 1998; 116-955-6.

23. Romano JJ, Iliff NT. Manson PN. Use of medpor porous poly ethylene implants in 140 patients with facial fractures. J Craniofacial Surgery 1993; 4:142.

24. Dougherty Wr, Wellisz T. the natural history of alloplasty implants in orbital floor reconstruction. An animal model. Craniofacial surgery 1994; 5:24.

25. Holmes R, Hagler H. Porous hydroxyapatite as a bone graft substitute in cranial reconstruction: A histometric study. Plast Reconstr Surgery 1988; 81:662.

26. Rubin PJ , Yaremchuck MJ . Complications and toxicities of implantable biomaterials used in facial aesthetics and reconstructive surgery. Plastic reconstructive surgery 1992; 100:1336.

27. Morrison AD, Sanderson RC, Moos KF. The use of silastic as an orbital implant for reconstruction of orbital wall defects: A review of 311 cases treated over 20 years. J Oral Maxillofacial Surgery 1995; 53:412. 